\title{
Effects of butylparaben on antioxidant enzyme activities and histopathological changes in rat tissues
}

\author{
Duygu Aydemir ${ }^{1,2}$, Burcu Öztaşc1 ${ }^{3}$, Nurhayat Barlas ${ }^{3}$, and Nuriye Nuray Ulusu ${ }^{1,2}$ \\ ${ }^{1}$ School of Medicine, Department of Medical Biochemistry, Koc University, Istanbul, Turkey \\ ${ }^{2}$ Koç University Research Center for Translational Medicine, Istanbul, Turkey \\ ${ }^{3}$ Department of Biology, Science Faculty, Hacettepe University, Ankara, Turkey
}

[Received in September 2019; Similarity Check in September 2019; Accepted in November 2019]

Butyl $p$-hydroxybenzoic acid, also known as butylparaben (BP), is one of the most common parabens absorbed by the skin and gastrointestinal tract and metabolised in the liver and kidney. Recent in vivo and in vitro studies have raised concern that BP causes reproductive, development, and teratogenic toxicity. However, BP-induced oxidative stress and its relation to tissue damage has not been widely investigated before. Therefore, we aimed to investigate the effects of butyl 4-hydroxybenzoate on enzyme activities related to the pentose phosphate pathway and on glutathione-dependent enzymes such as glucose 6-phosphate dehydrogenase (G6PD), 6-phosphogluconate dehydrogenase (6-PGD), glutathione reductase (GR), glutathione peroxidase (GPx), and glutathione-S-transferase (GST) in kidney, liver, brain, and testis tissues. Male rats were randomly divided into four groups to orally receive corn oil (control) or 200,400, or $800 \mathrm{mg} / \mathrm{kg} /$ day of BP for 14 days. Then we measured G6PD, GR, GST, 6-PGD, and GPx enzyme activities in these tissues and studied histopathological changes. BP treatment caused imbalance in antioxidant enzyme activities and tissue damage in the liver, kidney, brain, and testis. These findings are the first to show the degenerative role of BP on the cellular level. The observed impairment of equivalent homeostasis and antioxidant defence points to oxidative stress as a mechanism behind tissue damage caused by BP.

KEY WORDS: endocrine disrupting chemicals, glucose 6-phosphate dehydrogenase; 6-phosphogluconate dehydrogenase; glutathione reductase; glutathione peroxidase; glutathione-S-transferase

Butylparaben (butyl 4-hydroxybenzoate, BP) from the paraben family has widely been used as antimicrobial/ antifungal preservative in cosmetic products, deodorants, toiletries, face creams, pharmaceuticals, toys, food, and beverages for more than 50 years $(1,2)$. Parabens are endocrine disruptors which impair natural metabolism of hormones by mimicking, interfering, and/or blocking them (3-6).

After exposure through skin, ingestion, or inhalation, $\mathrm{BP}$ is metabolised by the liver, kidney, and skin esterases. Conjugated forms of BP are excreted in the urine and bile in humans, but some of it remains unchanged and accumulates in the body over time $(2,7-9)$. This accumulation raises health concerns, as paraben has been reported for oestrogen-like activity, endocrine disruption, and impairment of the reproductive system. Parabens are also closely associated with carcinogenesis, obesity, diabetes, inflammatory diseases, and neurodegenerative disorders $(10-15)$.

Molecular mechanisms behind this association remain unclear, but some studies have shown that endocrine

Corresponding author: Nuriye Nuray Ulusu, Koç University School of Medicine, Rumelifeneri Yolu, Sarıyer, 34450 Istanbul, Turkey

E-mail:nulusu@ku.edu.tr disrupting chemicals can damage tissue through oxidative imbalance that results in the damage of lipids, DNA, and proteins, which in turn triggers the development of a number of diseases $(16,17)$.

Antioxidant enzymes 6-phosphate dehydrogenase (G6PD), 6-phosphogluconate dehydrogenase (6-PGD), glutathione-S-transferase (GST), glutathione peroxidase (GPx), and glutathione reductase (GR) have a vital role in reductive biosynthesis and cellular detoxification from free radicals and chemicals to help the organism deal with internal and external stress $(4,18)$. GST, GR, and GPx are glutathione-dependent antioxidant enzymes, whereas G6PD and 6-PGD are glutathione-independent. G6PD is the ratelimiting enzyme in the pentose phosphate pathway (PPP) that maintains the cytosolic NADPH pool involved in cellular redox balance $(19,20)$. 6-PGD is another enzyme in the PPP pathway responsible for NADPH production. Their impairment is closely associated with elevated reactive oxygen species (ROS) in several diseases, including cancer, diabetes, inflammatory diseases, and obesity (16, $17,21,22)$. Reduced glutathione (GSH) is mainly responsible for the protection of cells against ROS and it is required as cofactor for glutathione dependent enzymes $(23,24)$ 
There are but a few studies on BP-induced oxidative stress in mice liver (25), human spermatozoa (26), and rat testis (27). None of them, however, reported BP-induced oxidative stress and tissue damage in the liver, kidney, or brain. Therefore, the aim of our study was to expand research of oxidative stress to these organs and histologically verify kidney, liver, brain, and testis tissue damage through oxidative stress as the mechanism behind BP, which may cause a number of abnormalities and diseases.

\section{MATERIALS AND METHODS}

\section{Materials}

Oxidised glutathione (GSSG), 6-phosphogluconate (6-PG), glucose-6-phosphate (G6P), nicotinamide adenine dinucleotide phosphate $\left(\mathrm{NADP}^{+}\right)$, reduced nicotinamide adenine dinucleotide phosphate $\left(\mathrm{NADPH}+\mathrm{H}^{+}\right)$, magnesium chloride $\left(\mathrm{MgCl}_{2}\right)$, butylparaben (butyl 4-hydroxybenzoate, $>99 \%$ purity), sodium phosphate monobasic and dibasic, tris, glutathione reductase (GR), hydrogen peroxide $\left(\mathrm{H}_{2} \mathrm{O}_{2}\right)$, ethylenediaminetetraacetic acid (EDTA), cOmplete ${ }^{\mathrm{TM}}$ Protease Inhibitor Cocktail, and sodium azide were obtained from Sigma-Aldrich (St. Louis, MO, USA). Alanine aminotransferase (ALT), aspartate aminotransferase (AST), urea, albumin, triglyceride, creatinine, and glucose kits for biochemical analysis were obtained from Audit Diagnostics (Cork, Ireland).

\section{Animal care and dose administration}

Male Wistar albino rats (Rattus norvegicus) were purchased from the Experimental Animals Production Center of Hacettepe University (Ankara, Turkey) at six weeks of age. We used 24 rats weighing 140-180 g. All were housed in polycarbonate cages with stainless steel covers in an air-conditioned room (12 h light/dark cycle with a temperature of $22 \pm 2{ }^{\circ} \mathrm{C}$ and relative humidity of $50 \pm 5 \%$ ). The animals were acclimated to the laboratory for one week before the experiments. During the 14-day BP administration, they had free access to tap water and standard rat pellet food (Korkutelim Feed Factory, Afyon, Turkey). All experimental procedures and animal use were approved by the Ethics Committee of Hacettepe University (B.30.2.HAC.0.05.0.6.00/132).

The value of low observed toxic effects (LOEL) for butylparaben is $1600 \mathrm{mg} / \mathrm{kg} /$ day (28). In our study, we selected halft the LOEL for our highest daily dose of $800 \mathrm{mg} / \mathrm{kg}$, and halved it further to 400 and $200 \mathrm{mg} / \mathrm{kg} /$ day. The rats were randomly divided into four groups of six to receive oil vehicle only (control) or 200, 400 or $800 \mathrm{mg} / \mathrm{kg}$ per day of BP solved in corn oil by daily oral gavage at nine clock a.m. for 14 consecutive days. On day 15, the animals were weighed and sacrificed under ether anaesthesia, followed by decapitation. Tissue samples, liver, kidney, brain and testis were removed and stored at $-80{ }^{\circ} \mathrm{C}$ until analysis.

\section{Serum biochemistry}

At the end of the experiment, all blood was taken from the heart of the rats with a sterile syringe and transferred to gel vacuum biochemistry tubes for serum analysis. Blood samples were then centrifuged at $500 \times g$ and $4{ }^{\circ} \mathrm{C}$ for 25 min to separate the serum fraction, which was then placed in Eppendorf tubes and stored at $-80^{\circ} \mathrm{C}$ until ALT, AST, urea, triglyceride, glucose, creatinine, and albumin analysis with a Schimadzu clinical spectrophotometer CL-770 (Kyoto, Japan) in the Toxicology Laboratory of Hacettepe University Department of Biology.

\section{Histopathological analysis}

Liver, kidney, testis, and brain samples of male rats were fixed in the Bouin's solution for $8 \mathrm{~h}$ before histopathological examination. All tissues were embedded in the paraffin, and $4 \mu \mathrm{m}$ thick sections stained with Harris haematoxylin and eosin (H\&E). All slides were inspected with an Olympus BX51 light microscope (200x magnification, (Tokyo, Japan). The photographs were processed with a Bs200prop software (BAB Imaging System, Ankara, Turkey).

\section{Tissue preparation and evaluation of enzyme activity}

All tissues were placed in ice-cold sterile physiological saline solution to wash out blood and then placed in liquid nitrogen.

Samples for determination of enzyme activity and protein concentrations were homogenised with an Ultra Turrax homogeniser with a S18N-10G probe (IKA, Königswinter, Germany) and mixed in a 1:3 volume with $50 \mathrm{mmol} / \mathrm{L}$ potassium phosphate buffer $(\mathrm{pH} 7.4)$ containing protease inhibitors. The homogenate was first centrifuged in a Beckman Coulter ultracentrifuge (Fullerton, CA, USA) at $105,000 \times \mathrm{g}$ at $4{ }^{\circ} \mathrm{C}$ for $60 \mathrm{~min}$, the pellet was removed, and the supernatants used to measure anti-oxidant enzyme activities with a LKB Ultraspec Plus spectrophotometer (4054 UV/visible, Biochrom Ltd., Cambridge, UK).

G6PD activity was measured according to the method described by Betke et al. (29) in a reaction mixture containing $0.6 \mathrm{mmol} / \mathrm{L} \mathrm{G6P}$ and $100 \mathrm{mmol} / \mathrm{L}$ of Tris-HCl buffer ( $\mathrm{pH} 8.0$ ) containing $10 \mathrm{mmol} / \mathrm{L} \mathrm{MgCl}_{2}$ and $0.2 \mathrm{mmol} / \mathrm{L} \mathrm{NADP}^{+}$. NADPH production was monitored at $340 \mathrm{~nm}$ at $37^{\circ} \mathrm{C}$ for $60 \mathrm{~s}$. Measurements were performed in duplicate, and one unit (U) of activity was defined as the amount of enzyme required to reduce one mmol $\mathrm{NADP}^{+} /$ min under the assay conditions. 6-phosphogluconate dehydrogenase activity (6PGD) was determined in the same way as above except that the mixture contained $0.6 \mathrm{mmol} / \mathrm{L}$ $6 \mathrm{PG}$ as substrate instead of G6P (30).

Glutathione reductase activity was determined according to a modified Staal method (31). Decrease in the absorbance of NADPH at $340 \mathrm{~nm}$ was monitored 
spectrophotometrically at $37^{\circ} \mathrm{C}$ for $60 \mathrm{~s}$. Unit of activity (U) was defined as the amount of enzyme needed to catalyse the oxidation of $1 \mu \mathrm{mol}$ of NADPH in $1 \mathrm{~min}$. GlutathioneS-transferase (GST) activity was evaluated by the determining the conjugation of reduced glutathione (GSH) with 1-chloro-2,4-dinitrobenzene (CDNB) (32). The assay mixture consisted of $0.2 \mathrm{~mol} / \mathrm{L}$ sodium phosphate buffer (pH 6.5), 20 mmol/L GSH, 20 mmol/L CDNB, $5 \mu \mathrm{L}$ tissue homogenate, and double distilled water added to fill up the volume to $500 \mu \mathrm{L}$. Experiments were carried out in the duplicate, and activities followed for $30 \mathrm{~s}$. Glutathione peroxidase (GPx) activity was measured according to Beutler et al (33). The assay mixture contained $100 \mathrm{mmol} / \mathrm{L}$ potassium phosphate buffer (pH 7.0), $200 \mathrm{mmol} / \mathrm{L}$ EDTA, GR enzyme $(10 \mathrm{U} / \mathrm{mL}), 400 \mathrm{mmol} / \mathrm{L}$ sodium azide, $100 \mathrm{mmol} / \mathrm{L}$ GSH, $2 \mathrm{mmol} / \mathrm{L}$ NADPH, $5 \mu \mathrm{L}$ tissue lysate, and distilled water to fill up the volume to $500 \mu \mathrm{L}$. The mixture was incubated at $37^{\circ} \mathrm{C}$ for $10 \mathrm{~min}$ and then $5 \mu \mathrm{L}$ of $10 \mathrm{mmol} / \mathrm{L} \mathrm{H}_{2} \mathrm{O}_{2}$ was added into the reaction mixture. The decrease in optical density (OD) of the system was measured at $340 \mathrm{~nm}$ at room temperature for $30 \mathrm{~s}$. One unit of activity (U) was defined the same as above.

Protein concentration of tissue homogenates was determined with the bicinchoninic (BCA) method in 96-well plates using Spectramax M2 microplate reader (BioTek, Winooski, VT, USA) (34).

\section{Statistical analysis}

For statistical analysis we used the GraphPad Prism software (San Diego, CA, USA). All data were analysed with one-way analysis of variance (ANOVA) followed by a Tukey's post hoc test for multiple comparison. The results were represented as mean \pm standard deviation (SD).

\section{RESULTS AND DISCUSSION}

\author{
Animal weight, food and water consumption
}

Table 1 shows the differences in food and water consumption and weight gain between control and BPtreated animal groups. Lower food consumption and correspondingly lower weight gain in BP-treated animals may point to adverse health effects of $\mathrm{BP}$.

\section{Tissue weight}

Table 2 shows differences in tissue weights between animal groups. Liver weight in the $200 \mathrm{mg} / \mathrm{kg}$ /day dose group was significantly lower and in the 400 and $800 \mathrm{mg} / \mathrm{kg} /$ day dose groups significantly higher than in controls. The last two groups also had significantly higher kidney weight than controls. These increases are indicative of tissue damage (35-37), which was confirmed by histopathological findings below. However, BP treatment did not affect the weights of the brain and testis.

\section{Serum biochemical parameters}

Table 3 shows that BP treatment significantly affected only ALT and AST levels. Low ALT and AST levels indicate impaired liver function $(36,37)$, which was confirmed by our histopathology and antioxidant enzyme findings below (Figure 2, Table 5).

\section{Antioxidant enzyme activities and histopathological confirmation}

Tables 4-7 clearly show that BP significantly affected anti-oxidant enzyme activities in rat liver, kidney, brain, and testis compared to control. This is in line with the few

Table 1 Food and water consumption of rats in the control and butylparaben-treated groups

\begin{tabular}{|c|c|c|c|c|}
\hline & \multirow{2}{*}{ Control } & \multicolumn{3}{|c|}{ Butylparaben } \\
\hline & & $200 \mathrm{mg} / \mathrm{kg} / \mathrm{day}$ & $400 \mathrm{mg} / \mathrm{kg} / \mathrm{day}$ & $800 \mathrm{mg} / \mathrm{kg} / \mathrm{day}$ \\
\hline Food consumption (g) & $14.61 \pm 2.82$ & $13.68 \pm 2.79$ & $12.51 \pm 1.43^{\mathrm{a}}$ & $13.88 \pm 1.66$ \\
\hline Water consumption $(\mathrm{ml})$ & $30.3 \pm 6.82$ & $28.07 \pm 5.36^{\mathrm{a}}$ & $32.24 \pm 3.63$ & $38.88 \pm 5.97^{\mathrm{a}, \mathrm{b}}$ \\
\hline Initial body weight (g) & $141.83 \pm 19.67$ & $147.83 \pm 10.14$ & $183.83 \pm 13.59$ & $188.83 \pm 7.29$ \\
\hline Final body weight (g) & $187 \pm 25.66$ & $182.67 \pm 17.75$ & $224.67 \pm 20.27$ & $232 \pm 17.81$ \\
\hline Weight gain (\%) & $31.39 \pm 11.83$ & $23.52 \pm 7.63^{\mathrm{a}}$ & $21.93 \pm 4.31^{\mathrm{a}}$ & $22.74 \pm 6.1^{\mathrm{a}}$ \\
\hline
\end{tabular}

All results are given as mean \pm SD of six animals. ${ }^{\text {a }}$ significantly different from control $(\mathrm{p} \leq 0.05)$

Table 2 Organ weight of rats in control and butylparaben-treated groups

\begin{tabular}{lcccc}
\hline \multirow{2}{*}{ Organ } & Control & \multicolumn{3}{c}{ Butylparaben } \\
\cline { 3 - 5 } & & $\mathbf{2 0 0} \mathbf{~} \mathbf{g} / \mathbf{k g} / \mathbf{d a y}$ & $\mathbf{4 0 0} \mathbf{~} \mathbf{g} / \mathbf{k g} / \mathbf{d a y}$ & $\mathbf{8 0 0} \mathbf{~} \mathbf{g} / \mathbf{k g} / \mathbf{d a y}$ \\
\hline Brain $(\mathrm{g})$ & $1.50 \pm 0.06$ & $1.59 \pm 0.08$ & $1.73 \pm 0.04$ & $1.72 \pm 0.05$ \\
\hline Liver $(\mathrm{g})$ & $7.5 \pm 1.02$ & $6.08 \pm 0.84^{\mathrm{a}}$ & $8.02 \pm 1.13^{\mathrm{b}}$ & $8.37 \pm 1.37^{\mathrm{a}, \mathrm{b}}$ \\
\hline Kidney $(\mathrm{g})$ & $1.44 \pm 0.22$ & $1.31 \pm 0.18$ & $1.56 \pm 0.19^{\mathrm{a}}$ & $1.7 \pm 0.22^{\mathrm{a}, \mathrm{b}}$ \\
\hline Testis $(\mathrm{g})$ & $1.28 \pm 0.12$ & $1.22 \pm 0.15$ & $1.40 \pm 0.14$ & $1.47 \pm 0.09$ \\
\hline
\end{tabular}

All results are given as mean \pm SD of six animals. ${ }^{a}$ significantly different from control; ${ }^{b}$ significantly different from the $200 \mathrm{mg} / \mathrm{kg} / \mathrm{day}$ dose group $(\mathrm{p} \leq 0.05)$ 
reports there are about BP-induced oxidative stress. The one by Shah and Verma (25) reported higher levels of oxidative stress enzymes GPx, GST, catalase (CAT), and superoxide dismutase (SOD) in mice liver. Samarasinghe et al. (26) have shown that parabens can induce oxidative stress in human spermatozoa. Schreiber et al. (27) reported that BP can impair oxidative stress metabolism in rat testis.

Furthermore, we have supported our results by histopathological findings indicating tissue damage (Figures 1-4).

Consistent changes have also been reported earlier by Zhang et al. (38), who found that $n$-butylparaben induced male reproductive disorders via deregulation of oestradiol and oestrogen receptors, reduced the layers of germinal epithelium, and changed the structure of the seminiferous tubule. Other authors reported that BP administration led to the apoptosis of spermatogenic cells in prepubertal rats and to the impairment of the Sertoli cell vimentin filaments
(38-40). Aubert et al. (41) suggested that tubular degeneration, tubular dilatation, and dead cells in the tubules found at the highest BP dose may have resulted from apoptosis to avoid irreversible DNA damage.

\section{CONCLUSION}

This is the first study to have investigated and clearly shown that BP can impair oxidative balance and cause damage across rat brain, kidney, and liver (in addition to the testis). These results are consistent with the degenerative role of butylparaben on the cellular level, as it impairs homeostasis and antioxidant defence.

Butyl 4-hydroxybenzoate may not be as safe as initially thought, as this chemical affects enzyme activity, cellular homeostasis, and key tissues. Our findings have pointed toward oxidative stress as a likely mechanism behind BPinduced damage, which calls for further investigation.
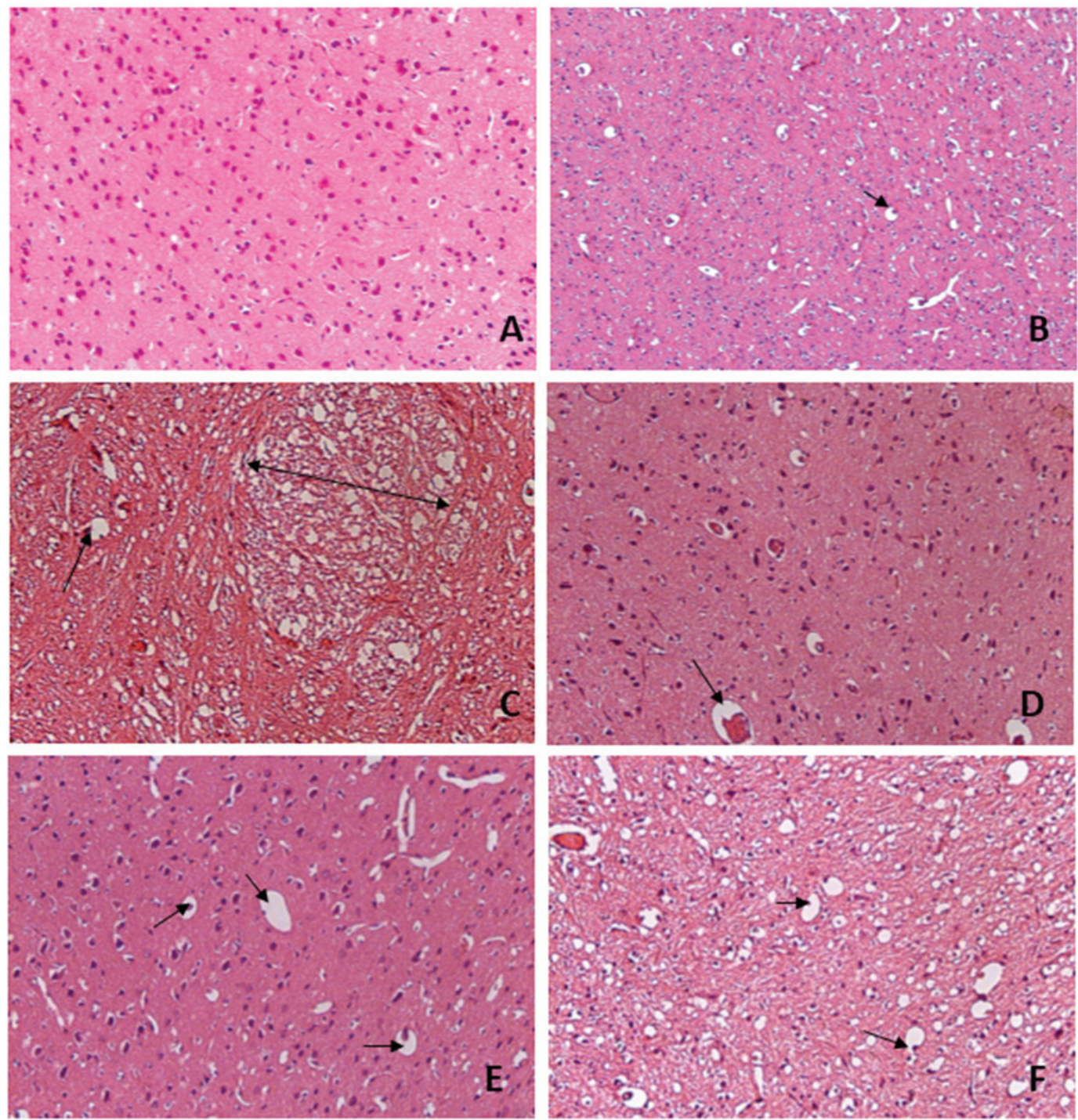

Figure 1 Photomicrographs showing hyperchromatic cells (marked with arrows) in the rat brain cortex of BP-treated groups (B - 200 $\mathrm{mg} / \mathrm{kg} / \mathrm{day} ; \mathrm{C}$ and D - $400 \mathrm{mg} / \mathrm{kg} / \mathrm{day} ; \mathrm{E}$ and F - $800 \mathrm{mg} / \mathrm{kg} /$ day) compared to control (A) (stained with H\&E, 200x magnification) 

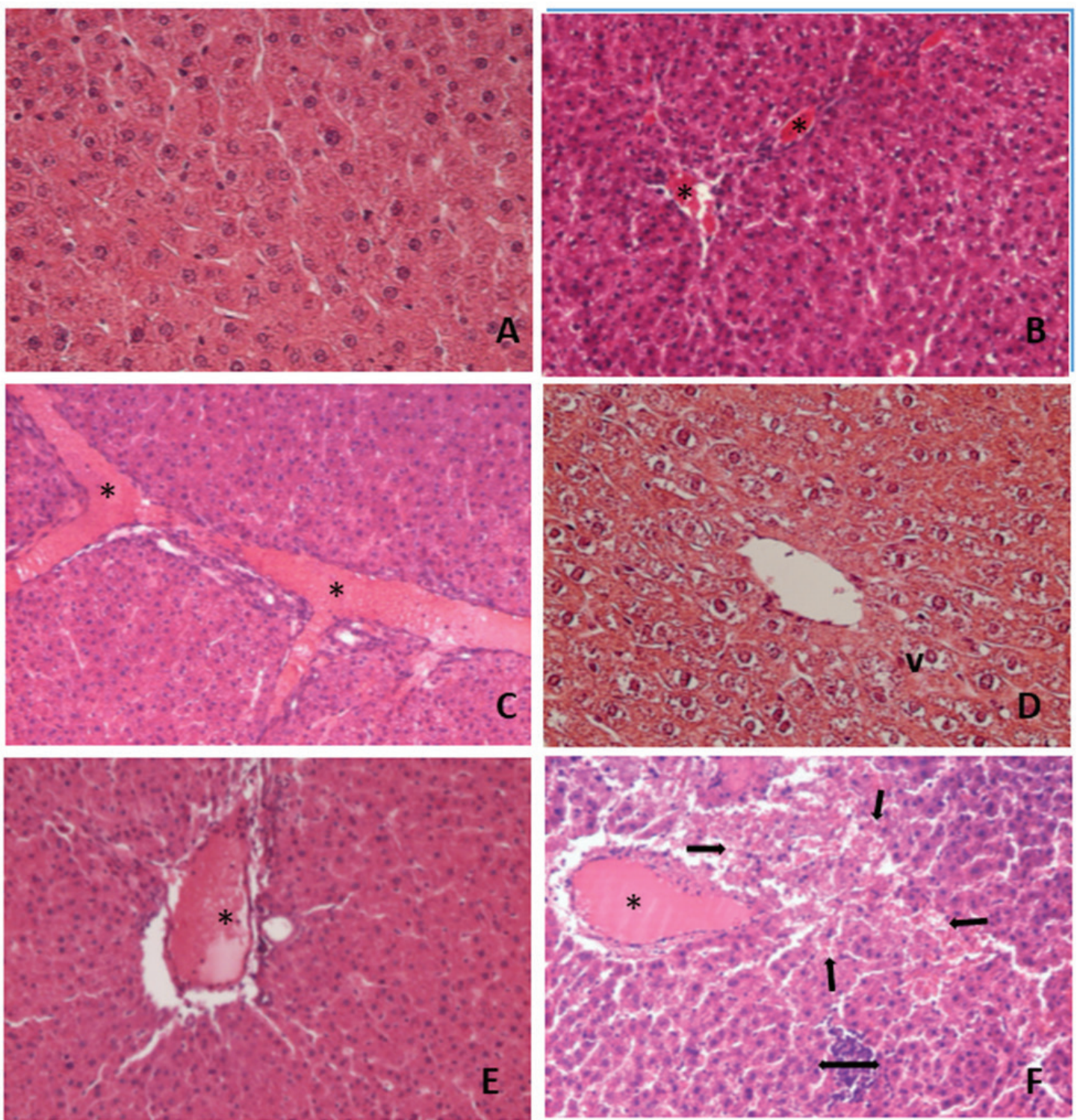

Figure 2 Photomicrographs showing congestion $(*)$, necrosis $(\rightarrow)$, mononucleer cell infiltration $(\leftrightarrow)$, and vacuolization $(v)$ of male rat liver tissue of the BP-treated groups (B - $200 \mathrm{mg} / \mathrm{kg} / \mathrm{day} ; \mathrm{C}$ and D - $400 \mathrm{mg} / \mathrm{kg} / \mathrm{day} ; \mathrm{E}$ and F $-800 \mathrm{mg} / \mathrm{kg} / \mathrm{day}$ ) compared to control (A) (stained with H\&E, 200x magnification)

Table 3 Serum biochemistry of control and butylparaben-treated groups

\begin{tabular}{|c|c|c|c|c|}
\hline \multirow{2}{*}{ Serum biochemical parameters } & \multirow{2}{*}{ Control } & \multicolumn{3}{|c|}{ Butylparaben } \\
\hline & & $200 \mathrm{mg} / \mathrm{kg} / \mathrm{day}$ & $400 \mathrm{mg} / \mathrm{kg} / \mathrm{day}$ & $800 \mathrm{mg} / \mathrm{kg} / \mathrm{day}$ \\
\hline ALT (UI/I) & $19.31 \pm 2.74$ & $17.1 \pm 0.85$ & $15.91 \pm 0.93^{*}$ & $17.05 \pm 1.05$ \\
\hline AST (UI/I) & $101.13 \pm 2.77$ & $83.52 \pm 3.76$ & $59.43 \pm 0.58 *$ & $60.8 \pm 0.42 *$ \\
\hline Glucose (mg/dL) & $179.82 \pm 16.04$ & $169.57 \pm 6.39$ & $166.28 \pm 17.49$ & $165.85 \pm 26.23$ \\
\hline Albumin $(\mathrm{g} / \mathrm{dL})$ & $2.78 \pm 0.14$ & $2.75 \pm 0.23$ & $2.9 \pm 0.17$ & $2.96 \pm 0.11$ \\
\hline Creatinine (mg/dL) & $0.15 \pm 0.04$ & $0.16 \pm 0.01$ & $0.15 \pm 0.02$ & $0.17 \pm 0.02$ \\
\hline Triglycerides $(\mathrm{mg} / \mathrm{dL})$ & $0.2 \pm 0.04$ & $0.21 \pm 0.05$ & $0.21 \pm 0.05$ & $0.2 \pm 0.04$ \\
\hline Urea (mg/dL) & $7.37 \pm 1.32$ & $7.68 \pm 1.43$ & $8.01 \pm 1.39$ & $8.07 \pm 1.39$ \\
\hline
\end{tabular}

All results are given as mean \pm SD of six animals. *significantly different from control $(\mathrm{p} \leq 0.05)$ 

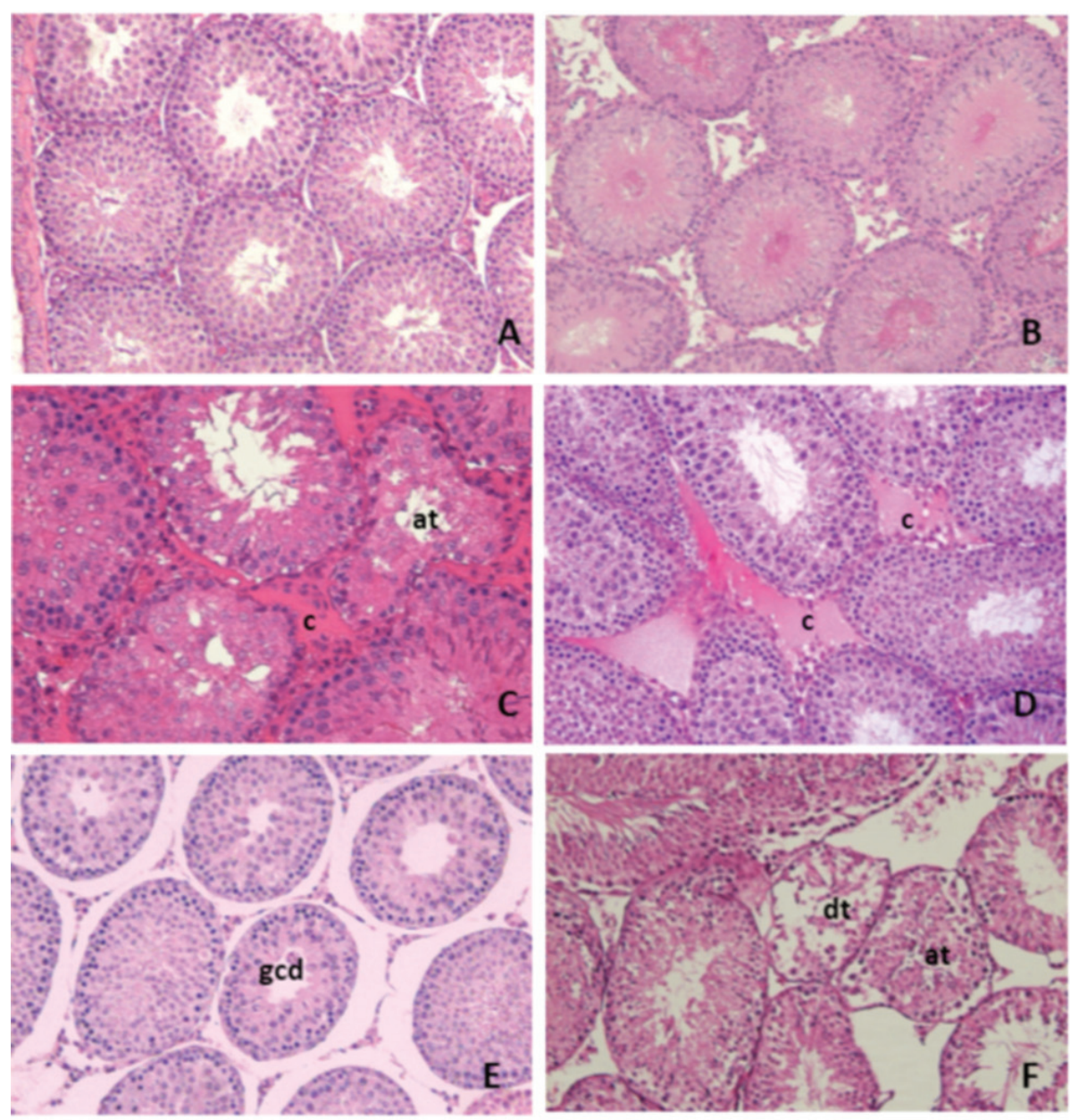

Figure 3 Photomicrographs showing atrophic tubules (at), congestion (c), germinal cell debris ( $\mathrm{gcd}$ ), and damaged tubules (dt) in testis tissues of the BP-treated groups (B - $200 \mathrm{mg} / \mathrm{kg} / \mathrm{day} ; \mathrm{C}$ and D - $400 \mathrm{mg} / \mathrm{kg} / \mathrm{day} ; \mathrm{E}$ and F $-800 \mathrm{mg} / \mathrm{kg} /$ day) compared to control (A) (stained with H\&E, 200x magnification)

Table 4 Brain antioxidant enzyme activities in control and butylparaben-treated rats

\begin{tabular}{|l|c|c|c|c|}
\hline \multirow{2}{*}{ Brain } & \multirow{2}{*}{ Control } & \multicolumn{3}{|c|}{ Butylparaben } \\
\cline { 3 - 5 } & $\mathbf{2 0 0} \mathbf{~ m g / k g / d a y ~}$ & $\mathbf{4 0 0} \mathbf{~ m g / k g / d a y ~}$ & $\mathbf{8 0 0} \mathbf{~ m g / k g / d a y ~}$ \\
\hline $\begin{array}{l}\text { Glucose-6-phosphate dehydrogenase } \\
\text { (G6PD) }\end{array}$ & $0.0561 \pm 0.008$ & $0.0771 \pm 0.012^{\mathrm{a}, \mathrm{b}, \mathrm{c}}$ & $0.1551 \pm 0.022^{\mathrm{d}, \mathrm{e}}$ & $0.0968 \pm 0.010^{\mathrm{f}}$ \\
\hline $\begin{array}{l}\text { 6-phosphogluconate dehydrogenase } \\
\text { (6PGD) }\end{array}$ & $0.0235 \pm 0.007$ & $0.0325 \pm 0.004^{\mathrm{f}}$ & $0.0654 \pm 0.012^{\mathrm{g}, \mathrm{h}}$ & $0.0415 \pm 0.003^{\mathrm{i}}$ \\
\hline Glutathione-S-transferase (GST) & $2.066 \pm 0.323$ & $0.9858 \pm 0.219^{\mathrm{j}, \mathrm{k}, \mathrm{l}}$ & $3.427 \pm 0.876^{\mathrm{m}, \mathrm{n}}$ & $1.699 \pm 0.22$ \\
\hline Glutathione reductase (GR) & $0.0394 \pm 0.007$ & $0.1297 \pm 0.023^{\mathrm{o}, \mathrm{p}}$ & $0.1460 \pm 0.019^{\mathrm{r}, \mathrm{s}}$ & $0.0670 \pm 0.007^{\mathrm{t}}$ \\
\hline Glutathione peroxidase (GPx) & $0.0725 \pm 0.018$ & $0.2066 \pm 0.083^{\mathrm{u}, \mathrm{v}, \mathrm{w}}$ & $0.6133 \pm 0.138^{\mathrm{y}, \mathrm{z}}$ & $0.2755 \pm 0.065$ \\
\hline
\end{tabular}

All results are given as mean \pm SD of $n=6$ animals. ${ }^{\text {a }} 200 \mathrm{mg} / \mathrm{kg} /$ day butylparaben dose group is different from control group ( $\left.\mathrm{p}=0.013\right) ;{ }^{b}$ $200 \mathrm{mg} / \mathrm{kg}$ /day dose group is different from $400 \mathrm{mg} / \mathrm{kg} /$ day dose group (p<0.0001); ${ }^{\mathrm{c}} 200 \mathrm{mg} / \mathrm{kg} /$ day dose group is different from $800 \mathrm{mg} / \mathrm{kg} / \mathrm{day}$ dose group ( $\mathrm{p}=0.024) ; \mathrm{d} 400 \mathrm{mg} / \mathrm{kg} /$ day dose group is different from control group ( $<<0.0001) ;{ }^{\mathrm{e}} 400 \mathrm{mg} / \mathrm{kg} /$ day dose group is different $800 \mathrm{mg} / \mathrm{kg} / \mathrm{day}$ group ( $\mathrm{p}<0.0001) ;{ }^{\mathrm{f}} 800 \mathrm{mg} / \mathrm{kg} /$ day dose group different from control group ( $\left.\mathrm{p}<0.0001\right) ; \mathrm{f}^{*} 200 \mathrm{mg} / \mathrm{kg} /$ day dose group is different from $400 \mathrm{mg} / \mathrm{kg} / \mathrm{day}$ group ( $\mathrm{p}<0.0001$ ); ${ }^{\mathrm{g}} 400 \mathrm{mg} / \mathrm{kg}$ /day butylparaben dose group is different from control group ( $\left.\mathrm{p}<0.0001\right) ;{ }^{\mathrm{h}} 400 \mathrm{mg} / \mathrm{kg} /$ day butylparaben dose group is different from $800 \mathrm{mg} / \mathrm{kg}$ /day dose group ( $\leq 0.0001) ;{ }^{i} 800 \mathrm{mg} / \mathrm{kg}$ /day dose group is different from control group ( $\mathrm{p}<0.0001$ ); ${ }^{j} 200 \mathrm{mg} / \mathrm{kg} /$ day butylparaben dose group is different from control group $(\mathrm{p}<0.0001) ;{ }^{\mathrm{k}} 200 \mathrm{mg} / \mathrm{kg} /$ day dose group is different from the $400 \mathrm{mg} / \mathrm{kg}$ /day dose group ( $<<0.0001) ;{ }^{1} 200 \mathrm{mg} / \mathrm{kg}$ /day dose group is different from $800 \mathrm{mg} / \mathrm{kg} /$ day dose group ( $\left.\mathrm{p}=0.014\right) ;{ }^{\mathrm{m}} 400 \mathrm{mg} / \mathrm{kg} / \mathrm{day}$ dose group is different from control group ( $\mathrm{p}<0.0001) ;{ }^{\mathrm{n}} 400 \mathrm{mg} / \mathrm{kg} /$ day dose group is different $800 \mathrm{mg} / \mathrm{kg} /$ day group (p<0.0001); ${ }^{\circ} 200 \mathrm{mg} / \mathrm{kg} /$ day butylparaben dose group is different from control group ( $<<0.0001$ ); ${ }^{\mathrm{p}} 200 \mathrm{mg} / \mathrm{kg} /$ day dose group is different from $800 \mathrm{mg} / \mathrm{kg}$ /day dose group ( $<<0.0001$ ); ${ }^{\mathrm{r}} 400 \mathrm{mg} / \mathrm{kg} /$ day dose group is different from control group ( $\left.\mathrm{p}=0.014\right) ;{ }^{\mathrm{s}} 400 \mathrm{mg} / \mathrm{kg} /$ day dose group is different from $800 \mathrm{mg} / \mathrm{kg} / \mathrm{day}$ dose group ( $\mathrm{p}<0.0001) ; \mathrm{t} 800 \mathrm{mg} / \mathrm{kg} /$ day dose group is different from control group $(\mathrm{p}<0.0001)$; ${ }^{\mathrm{a}} 200 \mathrm{mg} / \mathrm{kg} /$ day butylparaben dose group is different from control group ( $\mathrm{p}<0.0001) ;{ }^{\mathrm{v}} 200 \mathrm{mg} / \mathrm{kg} /$ day dose group is different from $400 \mathrm{mg} / \mathrm{kg} /$ day dose group ( $\left.\mathrm{p}<0.0001\right) ;{ }^{\mathrm{w}} 200 \mathrm{mg} / \mathrm{kg} /$ day dose group is different from $800 \mathrm{mg} / \mathrm{kg} /$ day dose group ( $\mathrm{p}=0.014$ ); ${ }^{y} 400 \mathrm{mg} / \mathrm{kg} /$ day dose group is different from control group ( $\left.<0.0001\right) ;{ }^{z} 400 \mathrm{mg} / \mathrm{kg} /$ day dose group is different $800 \mathrm{mg} / \mathrm{kg} /$ day group $(\mathrm{p}<0.0001)$ 

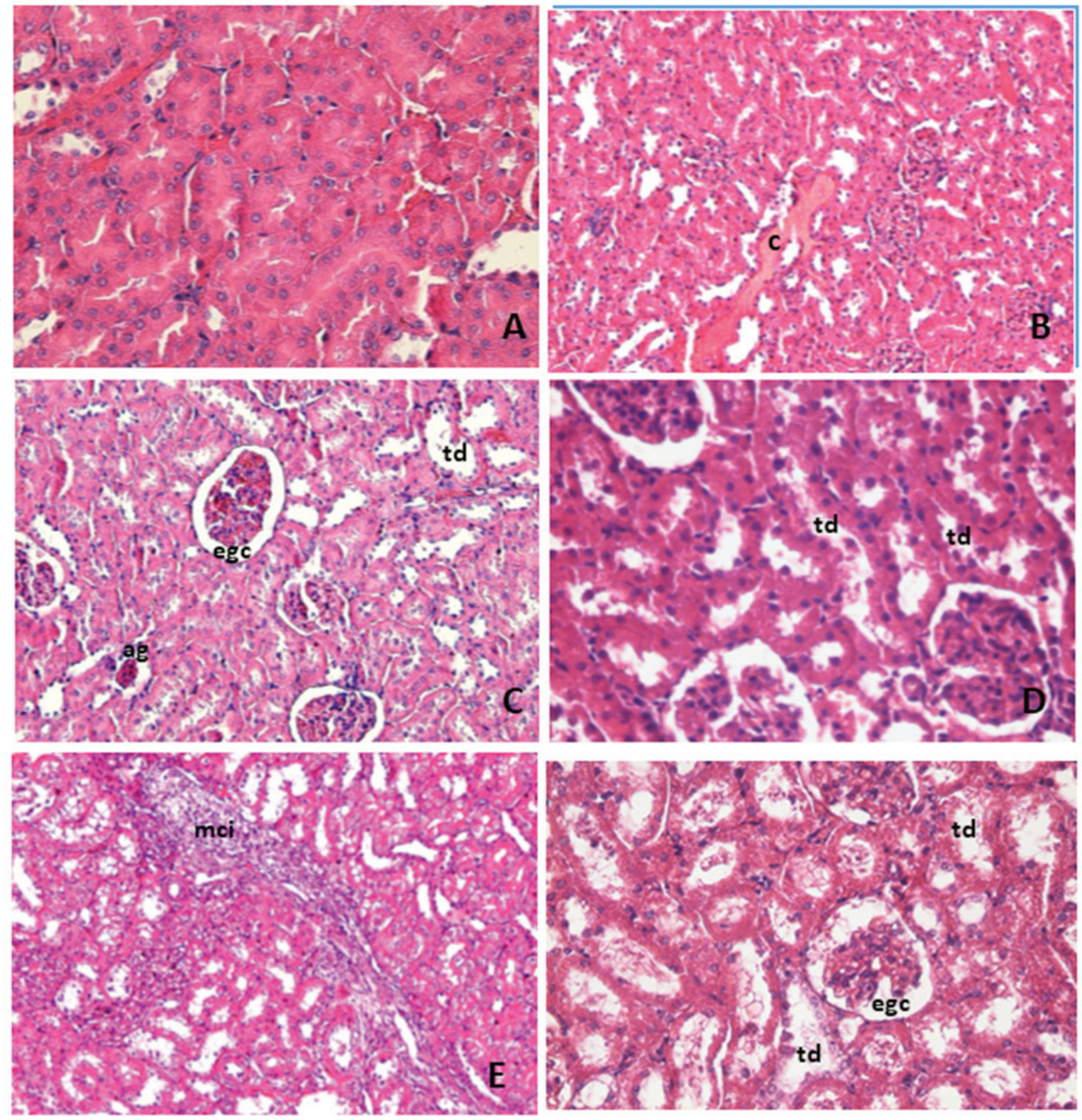

Figure 4 Photomicrographs of atrophic glomerulus (ag), congestion (c), enlargement of glomerular capsule (egc), tubular degeneration (td), and mononuclear cell infiltration (mci) in the kidney tissue the BP-treated groups (B - $200 \mathrm{mg} / \mathrm{kg} / \mathrm{day} ; \mathrm{C}$ and D $-400 \mathrm{mg} / \mathrm{kg} / \mathrm{day}$; $\mathrm{E}$ and $\mathrm{F}-800 \mathrm{mg} / \mathrm{kg} / \mathrm{day}$ ) compared to control (A) (stained with H\&E, 200x magnification)

Table 5 Liver antioxidant enzyme activities in control and butylparaben-treated rats

\begin{tabular}{|c|c|c|c|c|}
\hline \multirow{2}{*}{ Liver } & \multirow{2}{*}{ Control } & \multicolumn{3}{|c|}{ Butylparaben } \\
\hline & & $200 \mathrm{mg} / \mathrm{kg} / \mathrm{day}$ & $400 \mathrm{mg} / \mathrm{kg} / \mathrm{day}$ & $800 \mathrm{mg} / \mathrm{kg} / \mathrm{day}$ \\
\hline $\begin{array}{l}\text { Glucose-6-phosphate dehydrogenase } \\
\text { (G6PD) }\end{array}$ & $0.0342 \pm 0.002$ & $0.0338 \pm 0.011$ & $0.0475 \pm 0.001^{\mathrm{a}}$ & $0.0375 \pm 0.007$ \\
\hline $\begin{array}{l}\text { 6-phosphogluconate dehydrogenase } \\
\text { (6PGD) }\end{array}$ & $0.0891 \pm 0.017$ & $0.0629 \pm 0.014^{\mathrm{b}}$ & $0.0755 \pm 0.009^{c}$ & $0.0668 \pm 0.012^{\mathrm{d}}$ \\
\hline Glutathione-S-transferase (GST) & $13.91 \pm 3.554$ & $7.927 \pm 0.671^{\mathrm{e}}$ & $8.481 \pm 2.602^{\mathrm{f}}$ & $7.280 \pm 3.342^{\mathrm{g}}$ \\
\hline Glutathione reductase (GR) & $0.1583 \pm 0.021$ & $0.0954 \pm 0.008^{\mathrm{h}}$ & $0.1371 \pm 0.023^{\mathrm{i}}$ & $0.1527 \pm 0.028^{\mathrm{j}}$ \\
\hline Glutathione peroxidase (GPx) & $0.2382 \pm 0.076$ & $0.2222 \pm 0.070$ & $0.3933 \pm 0.117^{\mathrm{k}, 1}$ & $0.3566 \pm 0.102$ \\
\hline
\end{tabular}

All results were given as mean \pm SD of $\mathrm{n}=6$ animals. ${ }^{a} 400 \mathrm{mg} / \mathrm{kg} /$ day butylparaben dose group is different from $200 \mathrm{mg} / \mathrm{kg} / \mathrm{day}$ group $(\mathrm{p}=0.038) ;{ }^{\mathrm{b}} 200 \mathrm{mg} / \mathrm{kg} /$ day butylparaben dose group is different control group $(\mathrm{p}<0.0001) ;{ }^{\mathrm{c}} 400 \mathrm{mg} / \mathrm{kg} /$ day dose group is different from control group ( $\mathrm{p}=0.047) ;{ }^{\mathrm{d}} 800 \mathrm{mg} / \mathrm{kg} /$ day dose group is different from control group ( $\left.\mathrm{p}=0.015\right),(\mathrm{p}=0.021) ;{ }^{\mathrm{e}} 200 \mathrm{mg} / \mathrm{kg} / \mathrm{day}$ butylparaben dose group is different control group $(\mathrm{p} \leq 0.0001) ;{ }^{\mathrm{f}} 400 \mathrm{mg} / \mathrm{kg} /$ day dose group is different from control group ( $\left.\mathrm{p}<0.0001\right)$; $\mathrm{g} 800 \mathrm{mg} / \mathrm{kg} /$ day dose group is different from control group $(\mathrm{p}<0.0001) ;{ }^{\mathrm{h}} 200 \mathrm{mg} / \mathrm{kg} /$ day butylparaben dose group is different control group ( $\mathrm{p} \leq 0.0001) ;{ }^{i} 400 \mathrm{mg} / \mathrm{kg}$ /day dose group is different from $200 \mathrm{mg} / \mathrm{kg} /$ day dose group $(\mathrm{p}<0.0001) ;{ }^{j} 800 \mathrm{mg} / \mathrm{kg} /$ day dose group is different from $200 \mathrm{mg} / \mathrm{kg} /$ day dose group $(\mathrm{p}<0.0001) ;{ }^{\mathrm{k}} 400 \mathrm{mg} / \mathrm{kg}$ /day butylparaben dose group is different control group ( $\mathrm{p}=0.031$ ); ${ }^{1} 400 \mathrm{mg} / \mathrm{kg} /$ day dose group is different from $200 \mathrm{mg} / \mathrm{kg} /$ day dose group ( $\left.\mathrm{p}=0.015\right)$ 
Table 6 Testis antioxidant enzyme activities in control and butylparaben-treated rats

\begin{tabular}{|c|c|c|c|c|}
\hline \multirow{2}{*}{ Testis } & \multirow{2}{*}{ Control } & \multicolumn{3}{|c|}{ Butylparaben } \\
\hline & & $200 \mathrm{mg} / \mathrm{kg} / \mathrm{day}$ & $400 \mathrm{mg} / \mathrm{kg} / \mathrm{day}$ & $800 \mathrm{mg} / \mathrm{kg} / \mathrm{day}$ \\
\hline $\begin{array}{l}\text { Glucose-6-phosphate dehydrogenase } \\
\text { (G6PD) }\end{array}$ & $0.0316 \pm 0.006$ & $0.0623 \pm 0.010^{\mathrm{a}, \mathrm{c}, \mathrm{d}}$ & $0.0868 \pm 0.009^{\mathrm{b}, \mathrm{e}}$ & $0.0293 \pm 0.002$ \\
\hline $\begin{array}{l}\text { 6-phosphogluconate dehydrogenase } \\
\text { (6PGD) }\end{array}$ & $0.0378 \pm 0.011$ & $0.0915 \pm 0.014^{\mathrm{fg}, \mathrm{h}}$ & $0.0516 \pm 0.008^{\mathrm{i}, \mathrm{j}}$ & $0.0351 \pm 0.004$ \\
\hline Glutathione-S-transferase (GST) & $12.69 \pm 2.043$ & $15.30 \pm 3.561^{\mathrm{k}}$ & $11.9 \pm 1.853$ & $13.42 \pm 2.314$ \\
\hline Glutathione reductase (GR) & $0.0215 \pm 0.003$ & $0.0445 \pm 0.009^{1, \mathrm{~m}, \mathrm{n}}$ & $0.0243 \pm 0.004$ & $0.0355 \pm 0.005^{\mathrm{op}}$ \\
\hline Glutathione peroxidase (GPx) & $0.0764 \pm 0.007$ & $0.0208 \pm 0.008^{\mathrm{r}, \mathrm{s}, \mathrm{t}}$ & $0.0332 \pm 0.006^{\mathrm{u}, \mathrm{v}}$ & $0.0778 \pm 0.013$ \\
\hline
\end{tabular}

All results were given as mean $\pm \mathrm{SD}$ of $\mathrm{n}=6$ animals. ${ }^{\text {a }} 200 \mathrm{mg} / \mathrm{kg} /$ day butylparaben dose group is different from control group (p<0.0001); ${ }^{\mathrm{b}} 400 \mathrm{mg} / \mathrm{kg}$ /day dose group is different from control group ( $\left.\mathrm{p}<0.0001\right) ;{ }^{\mathrm{c}} 200 \mathrm{mg} / \mathrm{kg} /$ day dose group is different from $400 \mathrm{mg} / \mathrm{kg} / \mathrm{day}$ dose group ( $<<0.0001$ ); ${ }^{d} 200 \mathrm{mg} / \mathrm{kg} /$ day dose group is different from $800 \mathrm{mg} / \mathrm{kg} /$ day dose group ( $<0.0001$ ); ${ }^{\text {e }} 400 \mathrm{mg} / \mathrm{kg} /$ day dose group is different $800 \mathrm{mg} / \mathrm{kg}$ /day group $(\mathrm{p}<0.0001) ;{ }^{\mathrm{f}} 200 \mathrm{mg} / \mathrm{kg} /$ day dose group is different from control group ( $<<0.0001$ ); ${ }^{g} 200 \mathrm{mg} / \mathrm{kg} /$ day butylparaben dose group is different from $400 \mathrm{mg} / \mathrm{kg} /$ day dose group ( $\left.<<0.0001\right) ;{ }^{\text {h }} 200 \mathrm{mg} / \mathrm{kg} /$ day butylparaben dose group is different from $800 \mathrm{mg} / \mathrm{kg} /$ day dose group ( $\leq 0.0001) ;{ }^{i} 400 \mathrm{mg} / \mathrm{kg}$ /day dose group is different from control group ( $\mathrm{p}=0.013$ ); $\mathrm{j} 400 \mathrm{mg} / \mathrm{kg} /$ day dose group is different from $800 \mathrm{mg} / \mathrm{kg} /$ day dose group $(\mathrm{p}=0.025) ; \mathrm{k} 400 \mathrm{mg} / \mathrm{kg} /$ day butylparaben dose group is different from $200 \mathrm{mg} / \mathrm{kg} /$ day dose group $(\mathrm{p}=0.020) ;{ }^{1} 200 \mathrm{mg} / \mathrm{kg} /$ day dose group is different from control group $(\mathrm{p}<0.0001)$; ${ }^{\mathrm{m}} 200 \mathrm{mg} / \mathrm{kg} /$ day butylparaben dose group is different from $400 \mathrm{mg} / \mathrm{kg} /$ day dose group $(\mathrm{p}<0.0001) ;{ }^{\mathrm{n}} 200 \mathrm{mg} / \mathrm{kg} /$ day butylparaben dose group is different from $800 \mathrm{mg} / \mathrm{kg} /$ day dose group ( $\mathrm{p}=0.06) ;{ }^{\circ} 800 \mathrm{mg} / \mathrm{kg} /$ day dose group is different from control group ( $\mathrm{p}<0.0001$ ); p $800 \mathrm{mg} / \mathrm{kg} /$ day dose group is different from $400 \mathrm{mg} / \mathrm{kg}$ /day dose group ( $\mathrm{p}=0.005$ ); ${ }^{\mathrm{r}} 200 \mathrm{mg} / \mathrm{kg} /$ day dose group is different from control group $(\mathrm{p}<0.0001) ;{ }^{\mathrm{s}} 200 \mathrm{mg} / \mathrm{kg} /$ day butylparaben dose group is different from $400 \mathrm{mg} / \mathrm{kg} /$ day dose group ( $\left.\mathrm{p}=0.240\right)$; ${ }^{\mathrm{t}} 200 \mathrm{mg} / \mathrm{kg} / \mathrm{day}$ butylparaben dose group is different from $800 \mathrm{mg} / \mathrm{kg} /$ day dose group $(\mathrm{p}<0.0001) ;{ }^{\circ} 800 \mathrm{mg} / \mathrm{kg} /$ day dose group is different from control group ( $<00.0001$ ); ${ }^{p} 800 \mathrm{mg} / \mathrm{kg} /$ day dose group is different from $400 \mathrm{mg} / \mathrm{kg} /$ day dose group ( $\mathrm{p}=0.005$ ); u $400 \mathrm{mg} / \mathrm{kg} /$ day butylparaben dose group is different from control group $(\mathrm{p}<0.0001) ;{ }^{\vee} 400 \mathrm{mg} / \mathrm{kg} /$ day dose group is different from $800 \mathrm{mg} / \mathrm{kg} /$ day dose group $(\mathrm{p}<0.0001)$

Table 7 Kidney antioxidant enzyme activities in control and butylparaben-treated rats

\begin{tabular}{|c|c|c|c|c|}
\hline \multirow{2}{*}{ Kidney } & \multirow{2}{*}{ Control } & \multicolumn{3}{|c|}{ Butylparaben } \\
\hline & & $200 \mathrm{mg} / \mathrm{kg} / \mathrm{day}$ & $400 \mathrm{mg} / \mathrm{kg} / \mathrm{day}$ & $800 \mathrm{mg} / \mathrm{kg} / \mathrm{day}$ \\
\hline $\begin{array}{l}\text { Glucose-6-phosphate dehydrogenase } \\
\text { (G6PD) }\end{array}$ & $0.0316 \pm 0.006$ & $0.0623 \pm 0.010^{\mathrm{a}, \mathrm{c}, \mathrm{d}}$ & $0.0868 \pm 0.009^{\mathrm{b}, \mathrm{e}}$ & $0.0293 \pm 0.002$ \\
\hline $\begin{array}{l}\text { 6-phosphogluconate dehydrogenase } \\
\text { (6PGD) }\end{array}$ & $0.0378 \pm 0.011$ & $0.0915 \pm 0.014^{\mathrm{f,g}, \mathrm{h}}$ & $0.0516 \pm 0.008^{\mathrm{i}, \mathrm{j}}$ & $0.0351 \pm 0.004$ \\
\hline Glutathione-S-transferase (GST) & $12.69 \pm 2.043$ & $15.30 \pm 3.561^{\mathrm{k}}$ & $11.9 \pm 1.853$ & $13.42 \pm 2.314$ \\
\hline Glutathione reductase (GR) & $0.0215 \pm 0.003$ & $0.0445 \pm 0.009^{1, \mathrm{~m}, \mathrm{n}}$ & $0.0243 \pm 0.004$ & $0.0355 \pm 0.005^{\mathrm{o}, \mathrm{p}}$ \\
\hline Glutathione peroxidase (GPx) & $0.0764 \pm 0.007$ & $0.0208 \pm 0.008^{\mathrm{r}, \mathrm{s}, \mathrm{t}}$ & $0.0332 \pm 0.006^{u, v}$ & $0.0778 \pm 0.013$ \\
\hline
\end{tabular}

All results were given as mean \pm SD of $\mathrm{n}=6$ animals. ${ }^{a} 200 \mathrm{mg} / \mathrm{kg} /$ day butylparaben dose group is different from control group ( $\left.\mathrm{p}<0.0001\right)$; b $400 \mathrm{mg} / \mathrm{kg} /$ day dose group is different from control group ( $<<0.0001$ ); ${ }^{\mathrm{c}} 200 \mathrm{mg} / \mathrm{kg} /$ day dose group is different from $400 \mathrm{mg} / \mathrm{kg} / \mathrm{day}$ dose group $(\mathrm{p}<0.0001) ;{ }^{\mathrm{d}} 200 \mathrm{mg} / \mathrm{kg} /$ day dose group is different from $800 \mathrm{mg} / \mathrm{kg} / \mathrm{day}$ dose group $(\mathrm{p}<0.0001) ;{ }^{\mathrm{e}} 400 \mathrm{mg} / \mathrm{kg} / \mathrm{day}$ dose group is different $800 \mathrm{mg} / \mathrm{kg}$ /day group $(\mathrm{p}<0.0001) ;{ }^{\mathrm{f}} 200 \mathrm{mg} / \mathrm{kg} /$ day dose group is different from control group ( $<<0.0001$ ); ${ }_{\mathrm{g}} 200 \mathrm{mg} / \mathrm{kg} /$ day butylparaben dose group is different from $400 \mathrm{mg} / \mathrm{kg} /$ day dose group $(\mathrm{p}<0.0001) ;{ }^{\text {h }} 200 \mathrm{mg} / \mathrm{kg} /$ day butylparaben dose group is different from $800 \mathrm{mg} / \mathrm{kg} /$ day dose group ( $\leq 0.0001) ;{ }^{i} 400 \mathrm{mg} / \mathrm{kg}$ /day dose group is different from control group ( $\mathrm{p}=0.013$ ); ${ }_{j} 400 \mathrm{mg} / \mathrm{kg} /$ day dose group is different from $800 \mathrm{mg} / \mathrm{kg} /$ day dose group ( $\left.\mathrm{p}=0.025\right) ;{ }^{\mathrm{k}} 400 \mathrm{mg} / \mathrm{kg} /$ day butylparaben dose group is different from $200 \mathrm{mg} / \mathrm{kg}$ /day dose group ( $\mathrm{p}=0.020) ;{ }^{1} 200 \mathrm{mg} / \mathrm{kg}$ /day dose group is different from control group ( $\left.\mathrm{p}<0.0001\right) ;{ }^{\mathrm{m}} 200 \mathrm{mg} / \mathrm{kg} / \mathrm{day}$ butylparaben dose group is different from $400 \mathrm{mg} / \mathrm{kg} /$ day dose group $(\mathrm{p}<0.0001) ;{ }^{\mathrm{n}} 200 \mathrm{mg} / \mathrm{kg} /$ day butylparaben dose group is different from $800 \mathrm{mg} / \mathrm{kg} /$ day dose group ( $\mathrm{p}=0.06$ ); ${ }^{\circ} 800 \mathrm{mg} / \mathrm{kg} /$ day dose group is different from control group ( $\mathrm{p}<0.0001$ ); ${ }^{\mathrm{p}} 800 \mathrm{mg} / \mathrm{kg} / \mathrm{day}$ dose group is different from $400 \mathrm{mg} / \mathrm{kg} /$ day dose group $(\mathrm{p}=0.005) ;{ }^{\mathrm{r}} 200 \mathrm{mg} / \mathrm{kg}$ /day dose group is different from control group ( $\mathrm{p}<0.0001$ ); ${ }^{s} 200 \mathrm{mg} / \mathrm{kg} /$ day butylparaben dose group is different from $400 \mathrm{mg} / \mathrm{kg} /$ day dose group ( $\mathrm{p}=0.240$ ); ${ }^{\mathrm{t}} 200 \mathrm{mg} / \mathrm{kg} / \mathrm{day}$ butylparaben dose group is different from $800 \mathrm{mg} / \mathrm{kg} /$ day dose group $(\mathrm{p}<0.0001) ;{ }^{\circ} 800 \mathrm{mg} / \mathrm{kg}$ /day dose group is different from control group ( $\left.\mathrm{p}<0.0001\right)$; p $800 \mathrm{mg} / \mathrm{kg} /$ day dose group is different from $400 \mathrm{mg} / \mathrm{kg} /$ day dose group ( $\mathrm{p}=0.005$ ); ${ }^{\text {u }} 400 \mathrm{mg} / \mathrm{kg} / \mathrm{day}$ butylparaben dose group is different from control group ( $\mathrm{p}<0.0001) ;{ }^{\mathrm{v}} 400 \mathrm{mg} / \mathrm{kg}$ /day dose group is different from $800 \mathrm{mg} / \mathrm{kg} /$ day dose group $(\mathrm{p}<0.0001)$ 


\section{Acknowledgements}

This research was supported by the Scientific Research Projects Coordination Unit of Hacettepe University (project number FHD-2015-6025). We gratefully acknowledge the use of the services and facilities of the Koc University Research Center for Translational Medicine (KUTTAM), funded by the Research Infrastructure Support Program of the Turkish Ministry of Development. Findings, opinions, or points of view expressed in this article do not necessarily represent the official position or policies of the Ministry of Development.

\section{Conflict of interests}

None to declare.

\section{REFERENCES}

1. Guo Y, Kannan K. A survey of phthalates and parabens in personal care products from the United States and its implications for human exposure. Environ Sci Technol 2013;47:14442-9. doi: 10.1021/es4042034

2. Boberg J, Axelstad M, Svingen T, Mandrup K, Christiansen S, Vinggaard AM, Hass U. Multiple endocrine disrupting effects in rats perinatally exposed to butylparaben. Toxicol Sci 2016;152:244-56. doi: 10.1093/toxsci/kfw079

3. Boberg J, Taxvig C, Christiansen S, Hass U. Possible endocrine disrupting effects of parabens and their metabolites. Reprod Toxicol 2010;30:301-12. doi: 10.1016/j. reprotox.2010.03.011

4. Aydemir D, Karabulut G, Şimşek G, Barlas N, Ulusu NN. Impact of the Di(2-ethylhexyl) phthalate administration on trace element and mineral levels in relation of kidney and liver damage in rats. Biol Trace Elem Res 2018;186:474-88. doi: 10.1007/s12011-018-1331-0

5. Guerra MT, Sanabria M, Leite GA, Borges CS, Cucielo MS, Anselmo-Franci JA, Foster WG, Kempinas WG. Maternal exposure to butyl paraben impairs testicular structure and sperm quality on male rats. Environ Toxicol 2017;32:127389. doi: 10.1002/tox.22323

6. Ozdemir E, Barlas N, Cetinkaya MA. Assessing the antiandrogenic properties of propyl paraben using the Hershberger bioassay. Toxicol Res 2018;7:235-43. doi: 10.1039/c7tx00319f

7. NIEHS - National Institute of Environmental Health Sciences. Endocrine Disruptors [displayed 26 November 2019]. Available at http://www.niehs.nih.gov/health/topics/ agents/endocrine/

8. Commission Regulation(EU) No 1004/2014 of 18 September 2014 amending Annex V to Regulation (EC) No 1223/2009 of the European Parliament and of the Council on cosmetic products [displayed 26 November 2019]. Available at: https:// op.europa.eu/en/publication-detail/-/publication/a22d39484545-11e4-a0cb-01aa75ed71a1/language-en

9. Commission Regulation (EU) No 358/2014 of 9 April 2014 amending Annexes II and V to Regulation (EC) No $1223 / 2009$ of the European Parliament and of the Council on cosmetic product [displayed 26 November 2019]. Available at https://eur-lex.europa.eu/legal-content/EN/TXT/ PDF/?uri=CELEX:32014R0358
10. Ye X, Kuklenyik Z, Needham LL, Calafat AM. Automated on-line column-switching HPLC-MS/MS method with peak focusing for the determination of nine environmental phenols in urine. Anal Chem 2005;77:5407-13. doi: 10.1021/ ac050390d

11. Darbre PD, Harvey PW. Paraben esters: review of recent studies of endocrine toxicity, absorption, esterase and human exposure, and discussion of potential human health risks. J Appl Toxicol 2008;28:561-78. doi: 10.1002/jat.1358

12. Janjua NR, Frederiksen H, Skakkebaek NE, Wulf HC, Andersson AM. Urinary excretion of phthalates and paraben after repeated whole-body topical application in humans. Int J A ndrol $2008 ; 31: 118-30$. do i : 10.1111/j.1365-2605.2007.00841.x

13. Błędzka D, Gromadzińska J, Wąsowicz W. Parabens from environmental studies to human health. Environ Int 2014;67:27-42. doi: 10.1016/j.envint.2014.02.007

14. Bellavia A, Chiu YH, Brown FM, Mínguez-Alarcón L, Ford JB, Keller M, Petrozza J, Williams L, Ye X, Calafat AM, Hauser R, James-Todd T. Urinary concentrations of parabens mixture and pregnancy glucose levels among women from a fertility clinic. Environ Res 2019;168:389-96. doi: 10.1016/j.envres.2018.10.009

15. Moos RK, Angerer J, Dierkes G, Brüning T, Koch HM. Metabolism and elimination of methyl, iso- and n-butyl paraben in human urine after single oral dosage. Arch Toxicol 2016;90:2699-709. doi: 10.1007/s00204-015-1636-0

16. Halliwell B, Gutteridge JMC, Cross CE. Free radicals, antioxidants, and human disease: where are we now? J Lab Clin Med 1992;119:598-620. PMID: 1593209

17. Gutteridge JMC. Free radicals in disease processes: a compilation of cause and consequence. Free Radic Res Com 1993;19:141-58. doi: 10.3109/10715769309111598

18. Bhattacharyya A, Chattopadhyay R, Mitra S, Crowe SE. Oxidative stress: an essential factor in the pathogenesis of gastrointestinal mucosal diseases. Physiol Rev 2014;94:329 54. doi: 10.1152 physrev.00040.2012

19. Ho H, Cheng M, Tsun-yee CD. Glucose-6-phosphate dehydrogenase - from oxidative stress to cellular functions and degenerative diseases. Redox Rep 2007;12:109-18. doi: 10.1179/135100007X200209

20. Ulusu NN. Curious cases of the enzymes. J Med Biochem 2015;34:271-81. doi: 10.2478/jomb-2014-0045

21. Toda S. Polyphenol content and antioxidant effects in herb teas. Chin Med 2011;2:29-31. doi: 10.4236/cm.2011.21005

22. López-Alarcóna C, Denicola A. Evaluating the antioxidant capacity of natural products: a review on chemical and cellular-based assays. Anal Chim Acta 2013;763:1-10. doi: 10.1016/j.aca.2012.11.051

23. Ozdemir S, Tandogan B, Ulusu NN, Turan B. Angiotensin II receptor blockage prevents diabetes-induced oxidative damage in rat heart. Folia Biol (Praha) 2009;55:11-6. PMID: 19445841

24. Louie B, Rajamahanty S, Pyo P, Choudhury M, Konno S. Mode of cytotoxic action of nephrotoxic agents: oxidative stress and glutathione-dependent enzyme. BJU Int 2010;105:264-8. doi: 10.1111/j.1464-410X.2009.08657.x

25. Shah KH, Verma RJ. Butyl p-hydroxybenzoic acid induces oxidative stress in mice liver- an in vivo study. Acta Pol Pharm 2011;68:875-9. PMID: 22125952

26. Samarasinghe SV, Krishnan K, Naidu R, Megharaj M, Miller K, Fraser B, Aitken RJ. Parabens generate reactive oxygen 
species in human spermatozoa. Andrology 2018;6:532-41. doi: 10.1111/andr.12499

27. Schreiber E, Garcia T, Sharma PR, Torrente M, Domingo JL, Gómeza M. Oxidative stress in testes of rats exposed to n-butylparaben. Food Chem Toxicol 2019;131:110573. doi: 10.1016/j.fct.2019.110573

28. Rodrigues C, Lok E, Nera E, Iverson F, Page D, Karpinski $\mathrm{K}$, Clayson DB. Short-term effects of various phenols and acids on the Fischer 344 male rat forestomach epithelium. Toxicology 1986;38:103-17. doi: 10.1016/0300483x(86)90176-9

29. Betke K, Brewer GJ, Kirkman HN, Luzzato N, Motulsky AG, Ramot B, Siniscalco M. Standardized method for G-6PD assay of haemolysates. Annex 2. WHO Tech Rep Ser 1967;366:30-2.

30. Pearse B, Rosemeyer M. 6-phosphogluconate dehydrogenase from human erythrocytes. Methods Enzymol 1975;41:220 6. doi: 10.1016/s0076-6879(75)41051-5

31. Staal GEJ, Visser J, Veeger C. Purification and properties of glutathione reductase of human erythrocytes. Biochim Biophys Acta 1969;185:39-48. doi: 10.1016/00052744(69)90280-0

32. Habig W, Pabst M, Jakoby W. Glutathione S-transferases. The first enzymatic step in mercapturic acid formation. J Biol Chem 1974;249:7130-9. PMID: 4436300

33. Beutler E. Red Cell Metabolism: A Manual of Biochemical Methods. New York (NY): Grune \& Stratton; 1971.

34. Thermo Fisher Scientific. Pierce ${ }^{\mathrm{TM}}$ BCA Protein Assay Kit [displayed 26 November 2019]. Available at https://www. thermofisher.com/order/catalog/product/23225
35. Wassef L, Kelly DJ, Gilbert RE. Epidermal growth factor receptor inhibition attenuates early kidney enlargement in experimental diabetes. Kidney Int 2004;66:1805-14. doi: 10.1111/j.1523-1755.2004.00955.x

36. Strnad P, Harada M, Siegel M, Terkeltaub RA, Graham RM, Khosla C, Omary MB. Transglutaminase 2 regulates mallory body inclusion formation and injury-associated liver enlargement. Gastroenterology 2007;132:1515-26. doi: 10.1053/j.gastro.2007.02.020

37. Giannini EG, Testa R, Savarino V. Liver enzyme alteration: a guide for clinicians. CMAJ 2005;172:367-79. doi: 10.1503/ cmaj.1040752

38. Zhang L, Ding S, Qiao P, Dong L, Yu M, Wang C, Zhang M, Zhang L, Li Y, Tang N, Chang B. Effects of n-butylparaben on steroidogenesis and spermatogenesis through changed E2 levels in male rat offspring. Environ Toxicol Pharmacol 2014;37:705-17. doi: 10.1016/j.etap.2014.01.016

39. Alam MS, Ohsako S, Kanai Y, Kurohmaru M. Single administration of butylparaben induces spermatogenic cell apoptosis in prepubertal rats. Acta Histochem 2014;116:47480. doi: $10.1016 /$ j.acthis.2013.10.006

40. Alam MS, Kurohmaru M. Disruption of Sertoli cell vimentin filaments in prepubertal rats: An acute effect of butylparaben in vivo and in vitro. Acta Histochem 2014;116:682-7. doi: 10.1016/j.acthis.2013.12.006

41. Aubert N, Ameller T, Legrand JJ. Systemic exposure to parabens: pharmacokinetics, tissue distribution, excretion balance and plasma metabolites of [14C]-methyl-, propyland butyl paraben in rats after oral, topical or subcutaneous administration. Food Chem Toxicol 2012;50:445-54. doi: 10.1016/j.fct.2011.12.045

\section{Djelovanje butilparabena na aktivnost antioksidacijskih enzima i histopatološke promjene u tkivima štakora}

Butil $p$-hidroksibenzoična kiselina, poznata i pod nazivom butilparaben (BP), najčešći je oblik parabena, koji se apsorbira putem kože i probavnoga sustava te razgrađuje u jetri i bubrezima. Najnovija istraživanja in vivo i in vitro upozoravaju na to da BP djeluje toksično na reproduktivne organe, plod (teratogeno djelovanje) i razvoj organizma. No dosad nije detaljno istražen oksidacijski stres koji on izaziva niti njegova povezanost s oštećenjem tkiva. Stoga je cilj ovoga istraživanja bio utvrditi djelovanje BP-a na aktivnost enzima koji sudjeluju u pentoza fosfatnom putu i onih ovisnih o glutationu poput glukoza-6-fosfat dehidrogenaze (G6PD), 6-fosfoglukonat dehidrogenaze (6-PGD), glutation reduktaze (GR), glutation peroksidaze (GPx) i glutation-S-transferaze (GST) u tkivu bubrega, jetre, mozga i testisa. U tu su svrhu muški štakori bili nasumce raspodijeljeni u četiri skupine: tri su skupine četrnaest dana primale oralne doze BP-a od 200, $400 \mathrm{ili} 800 \mathrm{mg} / \mathrm{kg}$ na dan, a kontrolna skupina kukuruzno ulje. Nakon tretmana životinje su žrtvovane i u njihovim su tkivima izmjerene aktivnosti G6PD, GR, GST, 6-PGD i GPx te su napravljene histopatološke pretrage tkiva. BP je poremetio ravnotežu enzimskih aktivnosti te doveo do oštećenja svih ispitanih vrsta tkiva. Ovo su prvi nalazi koji potvrđuju degenerativnu ulogu BP-a na staničnoj razini. Zabilježeni poremećaji homeostaze i obrambenoga antioksidacijskoga sustava upućuju na oksidacijski stres kao mehanizam u podlozi oštećenja tkiva izazvanoga BP-om.

KLJUČNE RIJEČI: endokrini disruptori, glukoza-6-fosfat dehidrogenaza; 6-fosfoglukonat dehidrogenaza; glutation peroksidaza; glutation reduktaza; glutation-S-transferaza 